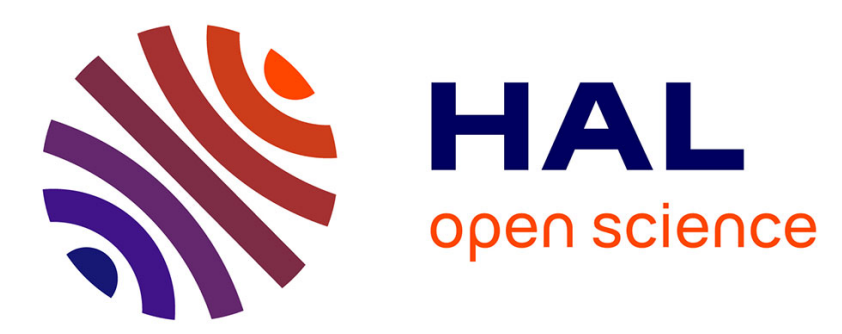

\title{
Vision-Based Force Sensing of a Magnetic Microrobot in a Viscous Flow
}

\author{
Karim Belharet, David Folio, Antoine Ferreira
}

\section{To cite this version:}

Karim Belharet, David Folio, Antoine Ferreira. Vision-Based Force Sensing of a Magnetic Microrobot in a Viscous Flow. IEEE International Conference on Robotics and Automation (ICRA'2014), May 2014, Hong Kong, China, France. pp.2065-2070, 10.1109/ICRA.2014.6907133 . hal-01070673

\section{HAL Id: hal-01070673 https://hal.science/hal-01070673}

Submitted on 2 Oct 2014

HAL is a multi-disciplinary open access archive for the deposit and dissemination of scientific research documents, whether they are published or not. The documents may come from teaching and research institutions in France or abroad, or from public or private research centers.
L'archive ouverte pluridisciplinaire HAL, est destinée au dépôt et à la diffusion de documents scientifiques de niveau recherche, publiés ou non, émanant des établissements d'enseignement et de recherche français ou étrangers, des laboratoires publics ou privés. 


\title{
Vision-Based Force Sensing of a Magnetic Microrobot in a Viscous Flow
}

\author{
Karim Belharet ${ }^{1}$, David Folio ${ }^{2}$ and Antoine Ferreira ${ }^{2}$
}

\begin{abstract}
This paper has presented a new vision-based force-sensing framework that allows to characterize the forces applied on a magnetic microrobot in an endovascular-like environment. Especially, unlike common approaches used with optical microscopy where orthographic projection model are used, we consider in this paper the weak-perspective model. The proposed vision-based force characterization allows to retrieve the three dimensional (3D) translational velocities and accelerations of a microrobot viewed from a digital microscope. Hence, thanks to the dynamic model the external forces are estimated on-line. The framework was applied and validated for a magnetic microrobot navigating in a viscous flow. Experimental results in two different environments illustrate the efficiency of the proposed method.
\end{abstract}

\section{INTRODUCTION}

Untethered microrobots can significantly improve many aspects of medicine and bioengineering by navigating through the cardiovascular networks to perform targeted diagnosis and therapy [1], [2]. In particular, the use of magnetic fields is till now the most considered approach, and different designs have been proposed in the literature [2], [3]. A first solution is to mimic microorganisms behavior using helical tail [4], [5] or elastic flagella [6] for bio-inspired magnetic swimming design. It has been shown that such designs are suitable in small vessels such as arterioles or capillaries, whereas in larger vessel (as arteries) bead pulling scheme is more efficient [7]. Indeed, bead pulling were successfully applied in the carotid artery of a living swine [8]. Thus, in this work we consider a spherical neodymium magnet as microrobot body (termed microrobot throughout the text). Nevertheless, whatever the propulsion scheme used, all contributions point out the problem of navigation controllability of microrobots in a viscous flow. Especially, all magnetic microrobot designs have to face important constraints related to the system dynamics. To improve the magnetic navigation strategy against the biological laws governing patient body, a characterization of the magnetic microrobot behavior within a microfluidic environment is mandatory.

Our motivation in this work is to characterize and validate the system's dynamic model of a magnetic microrobot navigating in a microfluidic viscous environment. To do so, the forces acting on the microrobot must be measured. Force measurements could be achieved using capacitive force sensors [9], atomic force microscope (AFM) [10], piezoresistive cantilevers [11], or magnetic bead [12]. But

\footnotetext{
${ }^{1} \mathrm{~K}$. Belharet is with Hautes Études d'Ingénieur campus Centre, PRISME EA 4229, Châteauroux, France.

${ }^{2}$ D. Folio and A. Ferreira are with INSA Centre Val de Loire, Université d'Orléans, PRISME EA 4229, Bourges, France. Corresponding author: David FOLIO (Email: david.foliodinsa-cvl.fr)
}

such approaches are intrusive, and it is troublesome to use them with the microrobot in endovascular-like environments. A non-intrusive solution is to consider vision-based forcesensing [13], [14]. Proposed methods usually rely on the measurement of the displacement or deformation retrieved from an imaging sensor. In our context, to ensure efficient navigation control of magnetic microrobot its location is determined from medical imaging such as magnetic resonance imaging (MRI) [15], or digital microscopy [16], [17]. Hence, no additional sensing modalities are required, and the vision sensor is a priori able to provide the force feedback [14]. Nevertheless, most vision-based force measurement techniques rely on elastically deformable objects properties. As in our case the considered object is a hard material in a viscous flow, such solutions seems limited.

The main contribution of the paper is to define a mapping between the system dynamics and sensory data acquired from an imaging system to characterize the endovascular like interaction forces applied on the magnetic microrobot. Classically, when dealing with an optical microscope the orthographic perspective model is considered, that is a scaling of the observed scene. However, pure orthographic projection is usually unrealistic, and methods that use orthographic projection are only valid in a limited domain where the distance and position effects can be neglected [18]. Therefore, we propose here to consider the weak-perspective model that is closer to the full perspective model, and allows to improve the knowledge of the external forces.

In the remainder of this paper, we first present in Sect. II a new vision-based force characterization based on the vision-based model. Then in Sect. III we apply the proposed approach to a magnetic microrobot navigating in a viscous flow. Sect. IV presents different experiments that illustrate the efficiency and robustness of the proposed framework, before to conclude in Sect. V.

\section{VISION-BASED FORCE MEASUREMENT}

The proposed vision-based force-sensing relies on the observation of the microrobot motion from an imaging sensor. To do so, we use the mapping between the system dynamics and the image data provided by the sensor-based model.

\section{A. The Sensor-Based Model}

We consider a fixed vision system observing a moving device (here the microrobot), and assuming that only the device motion imply a sensor signal variation, the sensorbased model is expressed as follows [19]:

$$
\dot{\mathbf{s}}=\mathbf{J}_{\boldsymbol{\xi}}(\mathbf{s}) \mathbf{v}_{0}
$$


where $\dot{\mathbf{s}}$ is the observed microrobot motion vector in the image acquired from the vision sensor, and $\mathbf{v}_{0}$ is the device velocity screw in the $3 \mathrm{D}$ Euclidean space expressed in the reference frame $\mathcal{F}_{0}=\left(O, \vec{x}_{0}, \vec{y}_{0}, \vec{z}_{0}\right)$ (see Fig. 1). The term $\mathbf{J}_{\boldsymbol{\xi}}(\mathbf{s})$ is the Jacobian matrix, often referred as image Jacobian [20]. The subscript $\boldsymbol{\xi}$ denotes that $\mathbf{J}_{\boldsymbol{\xi}}(\mathbf{s})$ is generally a function of the extrinsic $\boldsymbol{\xi}_{\mathrm{Ex}}$ and intrinsic $\boldsymbol{\xi}_{\text {In }}$ parameters of the sensor, and the tracked sensor features s. Indeed, the image Jacobian matrix could be decomposed as follows:

$$
\mathbf{J}_{\boldsymbol{\xi}}(\mathbf{s})=\mathbf{L}\left(\boldsymbol{\xi}_{\mathrm{In}}, \mathbf{s}\right) \cdot{ }^{c} \mathbf{W}_{0}\left(\boldsymbol{\xi}_{\mathrm{Ex}}\right)
$$

where $\mathbf{L}\left(\boldsymbol{\xi}_{\text {In }}, \mathbf{s}\right)$ is often referred as the interaction matrix [19], and the matrix ${ }^{c} \mathbf{W}_{0}\left(\boldsymbol{\xi}_{\mathrm{Ex}}\right)$ allows to transform the motion $\mathbf{v}_{0}$ of the device between here the sensor frame $\mathcal{F}_{c}$ and the reference frame $\mathcal{F}_{0}$, as illustrated in Fig. 1 .

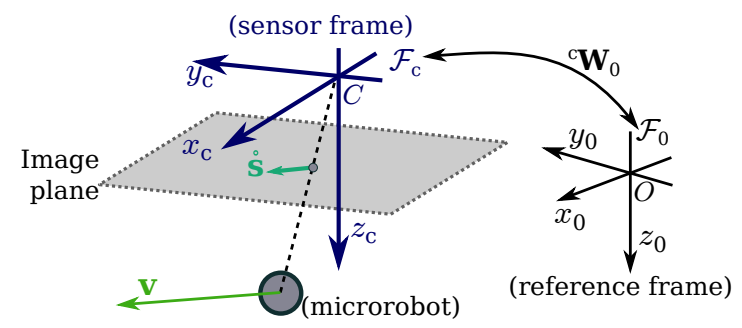

Fig. 1. Sensor-based modeling.

Let us assume that the image Jacobian matrix $\mathbf{J}_{\boldsymbol{\xi}}(\mathbf{s})$ is a full rank matrix, and then define $\mathbf{J}_{\boldsymbol{\xi}}^{+}=\left(\mathbf{J}_{\boldsymbol{\xi}}^{\top} \mathbf{J}_{\boldsymbol{\xi}}\right)^{-1} \mathbf{J}_{\boldsymbol{\xi}}^{\top}$ its Moore-Penrose pseudo-inverse. Thus, the previous visionbased model equation (1) could be re-written as follow:

$$
\mathbf{v}_{0}=\mathbf{J}_{\boldsymbol{\xi}}^{+} \dot{\mathbf{s}}
$$

This relation allows to characterize the microrobot motion in the $3 \mathrm{D}$ Euclidean space using vision data.

\section{B. Linking Vision-based Sensing to System Dynamics}

Now let us differentiate the vision-based model (1) to expose the sensor features dynamics:

$$
\ddot{\mathbf{s}}=\mathbf{J}_{\boldsymbol{\xi}}(\mathbf{s}) \dot{\mathbf{v}}_{0}+\mathbf{v}_{0} \cdot \mathbf{H}_{\boldsymbol{\xi}}(\mathbf{s}) \cdot \mathbf{v}_{0}
$$

where $\mathbf{H}_{\boldsymbol{\xi}}(\mathbf{s})$ is the image Hessian, defined as:

$$
\mathbf{H}_{\boldsymbol{\xi}}(\mathbf{s})=\frac{\partial \mathbf{J}_{\boldsymbol{\xi}}(\mathbf{s})}{\partial \mathbf{s}} \mathbf{J}_{\boldsymbol{\xi}}(\mathbf{s})=\mathbf{G}(\mathbf{s}) \mathbf{J}_{\boldsymbol{\xi}}(\mathbf{s})
$$

Substituting equation (3) into (4) yields:

$$
\dot{\mathbf{v}}_{0}=\mathbf{J}_{\boldsymbol{\xi}}^{+}\left(\ddot{\mathbf{s}}-\mathbf{G}(\mathbf{s}) \dot{\mathbf{s}} \mathbf{J}_{\xi}^{+} \dot{\mathbf{s}}\right)
$$

This relation allows to characterize the microrobot acceleration $\dot{\mathbf{v}}_{0}$ in the $3 \mathrm{D}$ Euclidean space, using the image feature $\mathrm{s}$ provided by the vision sensor.

Finally, thanks to the Newton's second law one can relate the microrobot acceleration $\dot{\mathbf{v}}_{0}$ to the forces acting on it:

$$
m \dot{\mathbf{v}}_{0}=\sum \mathbf{f}
$$

where $m$ is the microrobot mass, and $\sum \mathbf{f}$ is the net force expressed in the reference frame $\mathcal{F}_{0}$.

\section{APPLICATION TO A MAGNETIC MICROROBOT NAVIGATING IN A VISCOUS FLOW}

\section{A. Dynamics Modeling}

The considered microrobot body immersed in a microfluidic environment is modeled by a magnetic microsphere as illustrated on Fig. 2. The microrobot environment is modeled by a 3D Euclidean space, and we denote by $\mathcal{F}_{0}$ its fixed reference frame. Actuated by external magnetic gradients $\nabla \mathbf{b}$ in a microfluidic environment, the microrobot will mainly experience the steering magnetic $\left(\mathbf{f}_{\mathrm{m}}\right)$, apparent weight $\left(\mathbf{f}_{g}\right)$, contact $\left(\mathbf{f}_{\mathrm{c}}\right)$, electrostatic $\left(\mathbf{f}_{\mathrm{e}}\right)$, van der Waals $\left(\mathbf{f}_{\mathrm{v}}\right)$ and hydrodynamic drag $\left(\mathbf{f}_{\mathrm{d}}\right)$ microforces that affect the microrobot's motion. The effects of these forces are explained in detail in [7]. Hence, the translational motion of the ferromagnetic microsphere is formulated as follows :

$$
m \dot{\mathbf{v}}_{0}=\sum \mathbf{f}=\mathbf{f}_{\mathrm{m}}+\underbrace{\mathbf{f}_{\mathrm{d}}+\mathbf{f}_{g}+\mathbf{f}_{\mathrm{v}}+\mathbf{f}_{\mathrm{e}}+\mathbf{f}_{\mathrm{c}}}_{\mathbf{f}_{\text {Vasc }}}
$$

where $\mathbf{v}_{0}$ is the translational velocity of the microrobot and $m$ its mass.
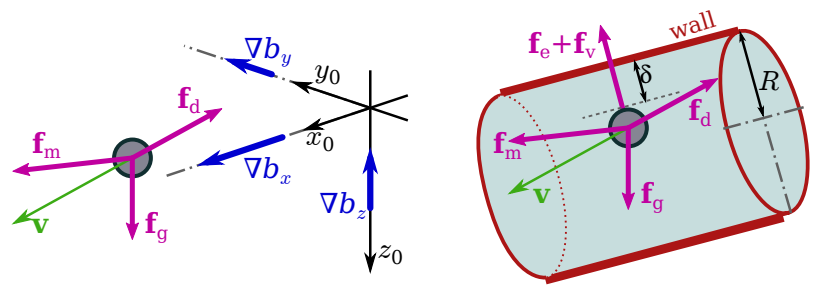

Fig. 2. Forces applied on a microrobot navigating in microfluidic environment: (left) in an infinite extend and (right) in cylindrical channel.

In the remainder of this paper, we assume that the orientation of the magnetic microrobot does not change due to the magnetic torque which tends to align the magnetization of the robot along the magnetic field. Hence, no angular motion is considered and the microrobot's velocity screw is reduced to its sole translational velocity: $\mathbf{v}_{0}=\left(v_{x}, v_{y}, v_{z}\right)^{\top}$. Moreover, we also assume that the microrobot is never in contact with the walls of the environment, namely $\mathbf{f}_{c}=0$. Actually, in case of wall contact, the vision-based force-sensing could be achieved using elastically deformable objects properties as in [13], [14].

\section{B. Digital Microscope Projection Model}

In this work, the magnetic microrobot motion is observed from a fixed digital microscope, and its position in the image plane is retrieved from image processing. As illustrated in Fig. 3, classically a 3D point of coordinates $\mathrm{x}=(X, Y, Z)^{\top}$ in the microscope frame $\mathcal{F}_{c}$ is projected into a $2 \mathrm{D}$ point with coordinates $\mathbf{s}_{p}=\left(x_{p}, y_{p}\right)^{\top}$ in the image plane with a pinhole perspective projection, and yields:

$$
x_{p}=f \frac{X}{Z}, \quad y_{p}=f \frac{Y}{Z}
$$

where $f$ is the focal length. If we denote $(u, v)$ the position of the corresponding pixel in the digitized image, this position 


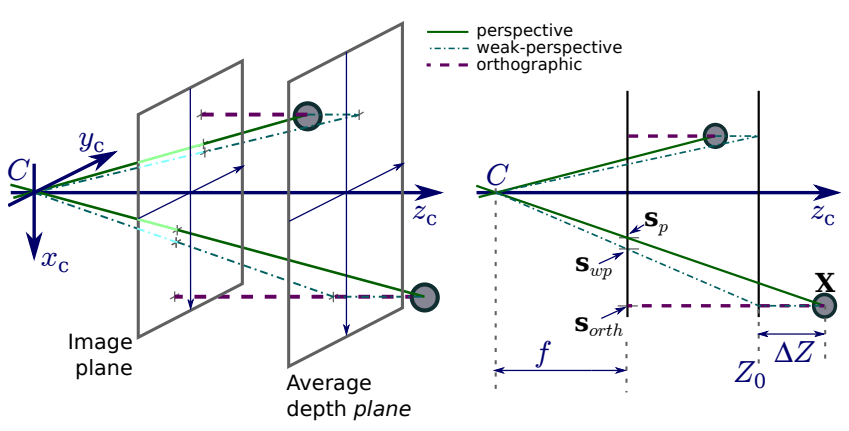

Fig. 3. Projection model: (a) 3D representation of image formation, and (b) full perspective $\left(\mathbf{s}_{p}\right)$, weak-perspective $\left(\mathbf{s}_{w p}\right)$ and orthographic perspective $\left(\mathbf{s}_{\text {orth }}\right)$ projection model comparisons.

is related to the $2 \mathrm{D}$ point $\mathrm{s}$ by:

$$
\left\{\begin{array}{l}
u=u_{0}+\alpha_{u} x_{p} \\
v=v_{0}+\alpha_{v} y_{p}
\end{array}\right.
$$

where $\alpha_{u}$ and $\alpha_{v}$ are the ratio between the focal length and the size of a pixel, and $\left(u_{0} v_{0}\right)$ is the principal point coordinate in pixel. Then, these four parameters define the digital microscope intrinsic parameters: $\boldsymbol{\xi}_{\text {In }}=\left\{\alpha_{u}, \alpha_{v}, u_{0}, v_{0}\right\}$, and are calibrated off-line [21], [22].

1) Orthographic perspective model: Generally, when a digital microscope is used, due to the size of the objects of interest wrt. the focal length $f$ and the vision system distance, the orthographic projection model is considered, that is:

$$
x_{\text {orth }}=k_{x} X, \quad y_{\text {orth }}=k_{y} Y
$$

where $k_{x}$ and $k_{y}$ scale the observed scene. As one can see, in orthographic projection, the depth $Z$ of the point $\mathbf{x}$ does not affect its image formation. However, in neglecting the depth information, the orthographic projection models image formation incorrectly and solves for (approximately) known parameters as if they were unknowns. It is given the freedom to reconstruct wrong values for these artificial unknowns, which in turn can corrupt the recovery of the true unknowns. Therefore, methods that use orthographic projection are only valid in a limited domain where the distance and position effects can be neglected.

Nevertheless, the full perspective projection model (9) requires a model or an estimation of the depth $Z$ of the considered 3D point $\mathbf{x}$. Several approaches may be used to determine it. The most obvious solution is to measure it using dedicated sensors such as telemeters or stereoscopic systems. However, if the system setup is not equipped with such sensors, it is possible to use structure from motion (SFM) techniques [23], signal processing methods [24], or even pose relative estimation [25]. Moreover, knowing an initial guess $Z\left(t_{0}\right)$, in [26] the authors propose to use the sensor-based model to estimate the $Z$-depth.

2) Weak perspective model: A suitable approximation is to use the so-called weak-perspective (or scaled orthographic) projection model, defined by:

$$
x_{w p}=f \frac{X}{Z_{0}}, \quad y_{w p}=f \frac{Y}{Z_{0}}
$$

where $Z_{0}$ is an average depth plane, as depicted in Fig. 3. The weak-perspective model is valid when the field of view is small and the average variation of the depth of the object $(\Delta Z)$ along the line of sight is small wrt. $Z_{0}$, i.e. $|\Delta Z| \ll$ $Z_{0}$. Especially, the weak-perspective is seen as a zero-order approximation of the full perspective projection (9). More precisely, for a point $\mathrm{x}$ of depth $Z=Z_{0}+\Delta Z$, the error is:

$$
\mathbf{s}_{e r r}=\mathbf{s}_{p}-\mathbf{s}_{w p}=-\frac{f}{Z_{0}} \frac{\Delta Z}{Z}\left[\begin{array}{c}
X \\
Y
\end{array}\right]
$$

This error shows that a small focal length $(f)$, small field of view $\left(X / Z_{0}\right.$ and $\left.Y / Z_{0}\right)$ and small depth variation $\Delta Z$ contribute to the validity of this model.

3) The interaction matrix: We have to use the interaction matrix $\mathbf{L}\left(\boldsymbol{\xi}_{\text {In }}, \mathbf{s}\right)$ that map visual feature motion $\dot{\mathbf{s}}$ to the microrobot velocity $\mathbf{v}_{0}(1)$. This matrix can be derived for many visual features, such as lines, circle, image moments, etc. [19]. In the case of a feature point $\mathbf{s}=(x, y)^{\top}$, the interaction matrix could be easily derived from the full projection model (9), and for a pure translational motion is given by:

$$
\mathbf{L}\left(\boldsymbol{\xi}_{\text {In }}, \mathbf{s}\right)=\left(\begin{array}{ccr}
\frac{f}{Z} & 0 & -\frac{x}{Z} \\
0 & \frac{f}{Z} & -\frac{y}{Z}
\end{array}\right)
$$

Using the weak-perspective, the above interaction matrix is then evaluated for the average plane $Z_{0}$. In this context the intrinsic parameters are defined by: $\boldsymbol{\xi}_{\text {In }}=\left\{\alpha_{u}, \alpha_{v}, u_{0}, v_{0}, Z_{0}\right\}$.

4) The transformation matrix: The transformation matrix ${ }^{c} \mathbf{W}_{0}\left(\boldsymbol{\xi}_{\mathrm{Ex}}\right)$ allows to transform the velocity screw from the camera frame $\mathcal{F}_{c}$ to the reference frame $\mathcal{F}_{0}$. In the case of a pure translational motion, this matrix is simply defined by:

$$
{ }^{c} \mathbf{W}_{0}\left(\boldsymbol{\xi}_{\mathrm{Ex}}\right)={ }^{c} \mathbf{R}_{0}
$$

where ${ }^{c} \mathbf{R}_{0} \in S O(3)$ (special orthogonal group of transformations of $\mathbb{R}^{3}$ ) is the rotation matrix between $\mathcal{F}_{c}$ and $\mathcal{F}_{0}$. As for intrinsic parameters $\boldsymbol{\xi}_{\text {In }}$, the transformation matrix parameters $\boldsymbol{\xi}_{\mathrm{Ex}}$ are calibrated off-line [21], [22].

\section{EXPERIMENTAL VALIDATION}

\section{A. Electromagnetic Actuation Testbed}

The motion control of the untethered microrobot in a microfluidic environment relies upon magnetic gradients $\nabla \mathbf{b}$. To this aim, an electromagnetic actuation (EMA) testbed has been developed specifically by Aeon-Scientific ${ }^{\mathrm{TM}}$ to generate the $3 \mathrm{D}$ controlled magnetic fields. The setup is illustrated in Fig. 4. The EMA setup consists of three nested sets of Maxwell coils and one nested set of Helmholtz coils. These coils set are combined coaxially such that the magnetic field and magnetic gradient fields can be controlled in the center of the workspace [17]. Hence, the magnetic gradient fields generated by the EMA system are controlled through the electrical currents circulating in the coil set.

Finally, the magnetic setup is equipped with a CCD high-resolution miniature microscope camera (TIMM 400, Nanosensor ${ }^{\mathrm{TM}}$ ) that is rigidly linked to the EMA setup. A robust tracking algorithm measure, with a sub-micrometer 


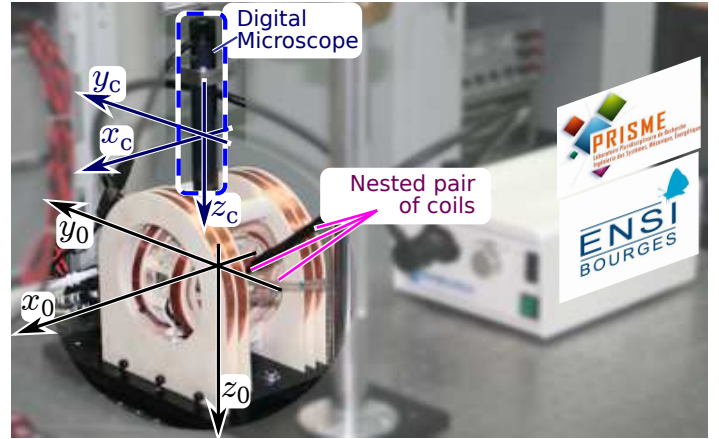

Fig. 4. Experimental testbed composed of four sets of EMA coils and a digital microscope.

resolution, the location of the magnetic microrobot by realtime images processing. Finally, after a calibration procedure, the extrinsic parameters are fully characterized such as the frame $\mathcal{F}_{0}$ and $\mathcal{F}_{c}$ are collinear.

\section{B. Experimentation Protocol}

In the experiments, a neodymium magnet ( $\mathrm{NdFeB} \mathrm{N35)}$ microsphere with a radius $r=250 \mu \mathrm{m}$ was used as microrobot body. To characterize the force net $\sum \mathbf{f}$ and validate the proposed vision-based force-sensing, experiments within different environments have been conducted (see Fig. 5). In particular, each experiment is realized within static viscous fluid made of a mixture of water and $80 \%$ of glycerol which is close to blood flow viscosity $\left(\eta_{f}=60 \mathrm{mPa} / \mathrm{s}\right)$. Each perspective model is calibrated twice: before the first experiments in i) free and ii) microfluidic channel environments. To facilitate the external force calibration a constant magnetic gradient is applied in the $x$-axis direction, and in the $z$-axis direction to compensate the gravitational force, leading to a straight line motion as depicted in Fig. 5.

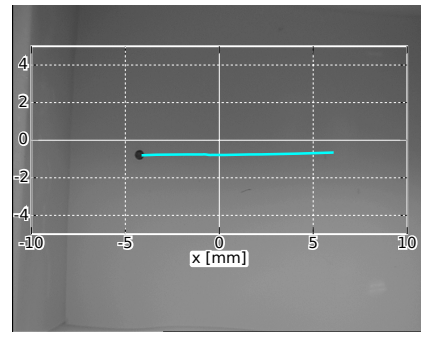

(a)

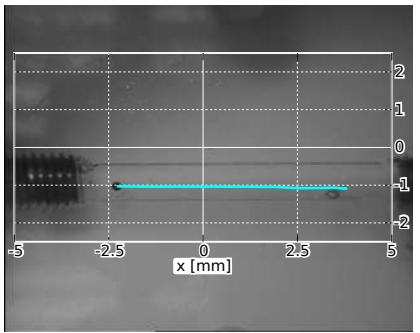

(b)
Fig. 5. Experiments in (a) free extend and (b) in a microchannel of radius $R=500 \mu \mathrm{m}$.

\section{Results in Free Extend}

First, experiments in a viscous fluid with "no walls" are performed to calibrate the velocity and the interaction forces without wall effects, as described in Fig. 5(a). Hence, DLVO forces (that is van der Waals and electrostatic forces) could be effectively neglected, and mainly the magnetic, the hydrodynamic and the gravitational forces could be considered in the interaction force expression (8). Within this free extend

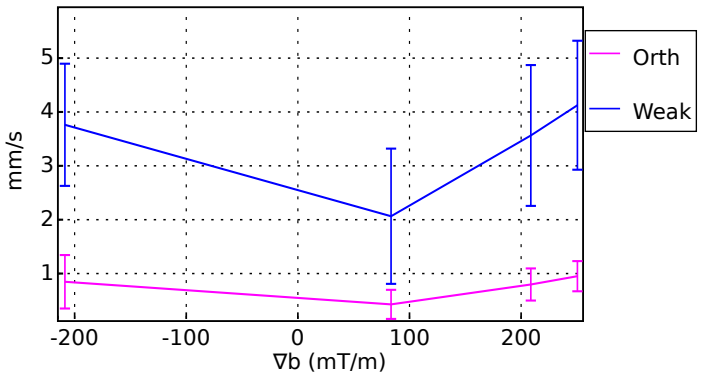

(a)

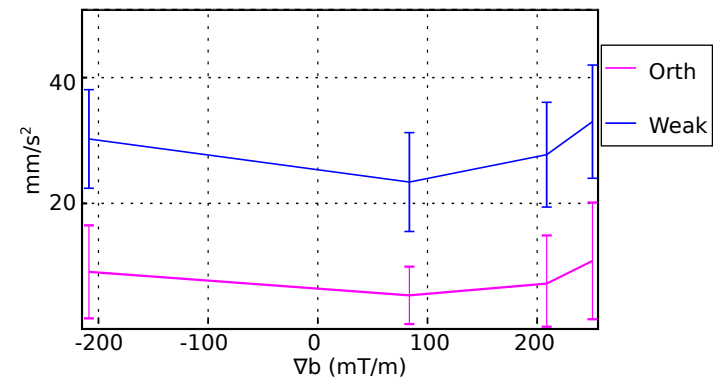

(b)

Fig. 6. Comparison of (a) the velocity $\mathbf{v}_{0}$ and (b) the acceleration $\dot{\mathbf{v}}_{0}$ of the orthographic perspective and the weak-perspective.

the average depth of the weak-perspective model is calibrated at $Z_{0}=67.67 \mathrm{~mm}$. Then, experiments are conducted with different magnetic gradients applied along the $x$-axis.

Fig. 6 illustrates the velocities and accelerations using the orthographic projection and the weak-perspective models with different magnetic gradients. As expected the velocities and accelerations decrease with the magnetic gradient amplitude. Furthermore, as the orthographic projection is less reliable, it tends to underestimate the velocity, implying a poor acceleration estimation. In particular, as the orthographic projection consider only the aspect ratio, only the 2D components $\left(v_{x}, v_{y}\right)$ of the microrobot's motion $\mathbf{v}_{0}$ could be directly estimated. Although the sole midplane $Z_{0}$ is considered, the weak-projection is able to provide an approximation of the full 3D motions. Actually, here the interaction matrix (14) is evaluated with the average plane $Z_{0}$.

Thus, knowing the microrobot motion in the free extend, the dynamics model introduced in Sect. III-A is computed (see [7] for detailed forces model). For instance, Fig. 7(a) shows the forces using the orthographic perspective and Fig. 7(b) using the weak-perspective model with a constant magnetic gradient $\nabla b_{x}=208.7 \mathrm{mT} / \mathrm{m}$ along the $x$-axis. Using a top view digital microscope, the orthographic model is capable to consider only the $x-y$ plane and through a scaling retrieved only the $2 \mathrm{D}$ components of the magnetic and hydrodynamic drag forces. Especially, by not explicitly considering some depth information, the orthographic projection models image formation incorrectly and mis-estimates the unknown parameters. In contrast, the weak-projection allows us to estimate the full 3D motions and dynamics. Therefore, thanks to our proposed framework we are able to consider the gravitational forces $\mathbf{f}_{g}$, and improve the force 


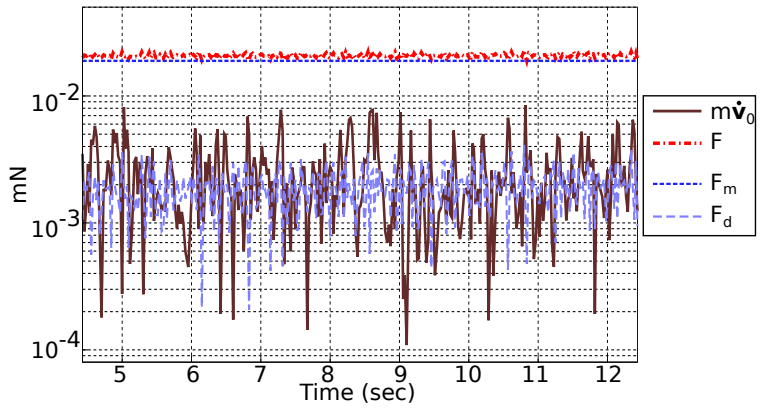

(a)

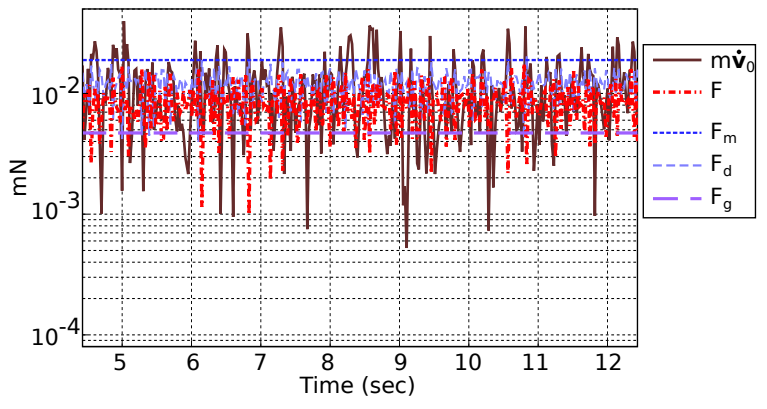

(b)

Fig. 7. Force model and magnetic microrobot's dynamics using (a) the orthographic perspective and (b) the weak-perspective models with $\nabla b_{x}=$ $208.7 \mathrm{mT} / \mathrm{m}$.

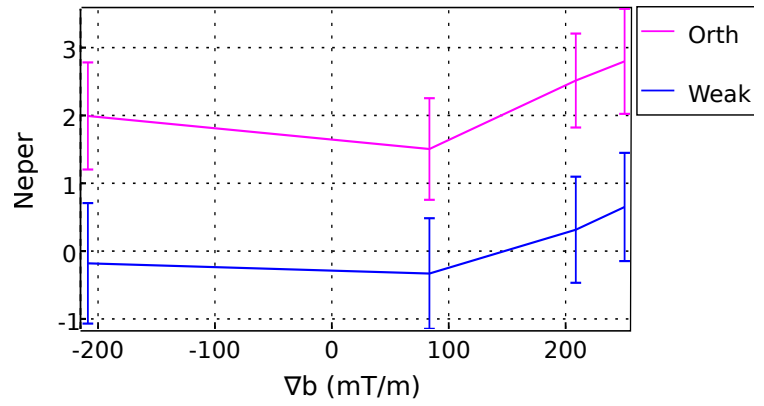

Fig. 8. Comparison between the force balance model and the microrobot dynamics in a free extend: $\log \left\|\sum \mathbf{f}\right\|-\log \left\|m \dot{\mathbf{v}}_{0}\right\|$.

balance model (8).

Hence, Fig. 8 presents the difference between the logarithmic error between the force net $\sum \mathbf{f}$ computed using the model referred in [7] and the microrobot acceleration computed from vision-based measurements, that is: $\log \left\|\sum \mathbf{f}\right\|-\log \left\|m \dot{\mathbf{v}}_{0}\right\|$, with different magnetic gradient amplitudes. As one can see, our framework seems to validate the proposed system's dynamic model. Furthermore, the use of weak perspective model allows to improve the knowledge of microrobot velocities and accelerations.

\section{Results within a microfluidic channel}

Secondly, experiments in a viscous fluid within a channel of radius $R=500 \mu \mathrm{m}$ are realized, as shown in Fig. 5(b). The average depth is here calibrated at $Z_{0}=85.26 \mathrm{~mm}$, and the distance to the wall is in average of $\delta=0.256 \mathrm{~mm}$. In such microfluidic environment, van der Waals forces remain negligible (as it was in the order of $10^{-14} \mathrm{mN}$ ) whereas

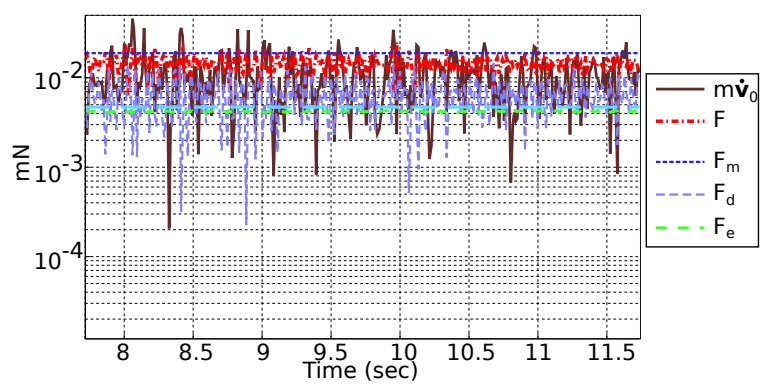

(a)

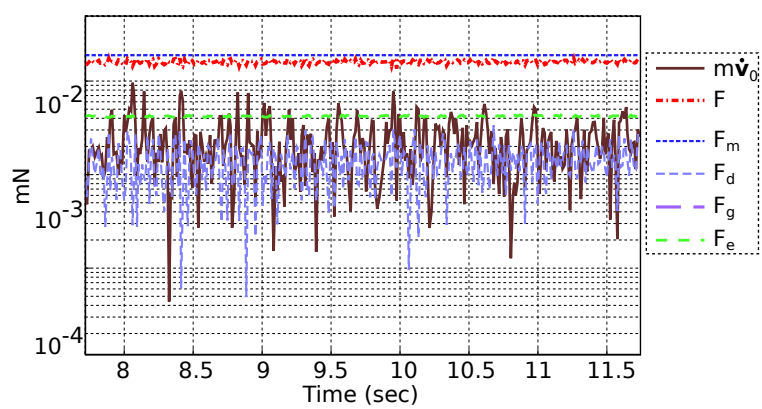

(b)

Fig. 9. Force model and magnetic microrobot's dynamics using (a) the orthographic perspective and (b) the weak-perspective models with $\nabla b_{x}=$ $208.7 \mathrm{mT} / \mathrm{m}$.

the electrostatic forces become significant, as illustrated on Fig. 9. Especially, Fig. 9(a) and 9(b) represent the forces computed from the orthographic perspective and the weak perspective, respectively. The results consider a constant magnetic gradient $\nabla b_{x}=208.7 \mathrm{mT} / \mathrm{m}$ along the $x$-axis. Once again, the orthographic projection is able to deal only with the $2 \mathrm{D}$ components of the force balance (8) in the $x-y$ plane, and does not allow to consider explicitly the gravitational forces along the $z$-axis.

Fig. 10 shows the logarithmic error between the force balance model (8) and the microrobot acceleration computed using our proposed approach based on the weak-perspective model. In particular the dashed line represents the logarithmic error when no electrostatic forces are considered, in opposition to the solid line. As one can see, adding the electrostatic forces knowledge may help to improve the dynamic model of the microrobot.

\section{E. Discussion}

The experimental results show that the proposed framework permits to characterize the external forces applied on a microrobot navigating in an endovascular-like environment from imaging data. Based only on scaling considerations, the orthographic model is not directly and explicitly able to deal with the $3 \mathrm{D}$ components of the motions nor dynamics. To get access to the 3D motions some knowledge on the $Z$ depth is mandatory. A first step is to use the average depth $Z_{0}$, and thus the weak perspective projection. This model allows to estimate the full 3D motion of the microrobot, and significantly improve the approximation of the microrobot 


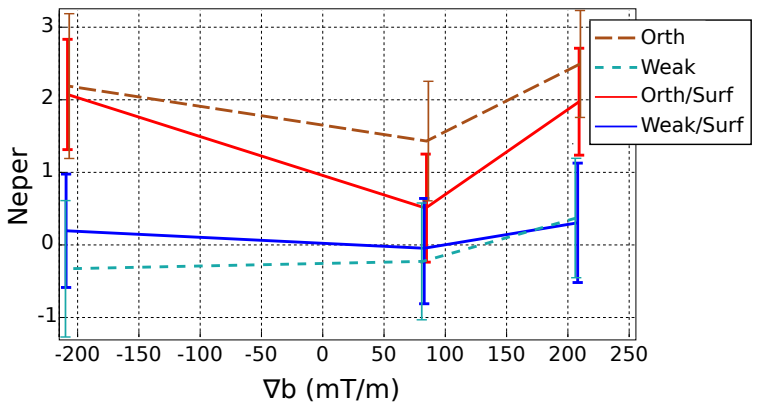

Fig. 10. Comparison between the force balance model and the microrobot dynamics in a microchannel: $\log \left\|\sum \mathbf{f}\right\|-\log \left\|m \dot{\mathbf{v}}_{0}\right\|$. Dashed-line without electrostatic forces, solid line with electrostatic forces.

motion. However, results also exhibit that the use of the third component increases the variance on the recovered 3D motion, and then, on the experimental forces. This behavior is due to the fact that the weak-perspective is based on the use of the average plane $Z_{0}$ instead of the true $Z$-depth of the microrobot. This issue could be overcame by using a second lateral digital microscope for instance, and thus consider the full perspective model.

\section{CONCLUSIONS}

This paper presented a new framework to characterize forces applied to a magnetic robot through the use of the vision-based and weak perspective models. To this aim a mapping between the vision-based data and the system's dynamic model is expressed. More precisely, unlike classical approach that uses an orthographic projection model when a microscope is considered, we have proposed here to deal with the weak-perspective model. In particular, the weakperspective is known to be closer to the full perspective. Hence, the proposed vision-based force sensing formalism allows to recover the full three dimensional motion and dynamics of the magnetic microrobot in order to characterize experimentally the external forces acting on the microrobot's body. Thus, the external forces could easily be characterized on-line from the images acquired by the digital microscope. The proposed method has been applied and experimentally validated for a magnetic microrobot navigating in a viscous flow. Finally, the experimental results illustrate the efficiency of the proposed framework, and validate the dynamics modeling of the swimming microrobot evolving in a free extend and in a microchannel. In both cases, the influence of the DLVO forces are demonstrated improving significantly the estimation of the force net.

\section{REFERENCES}

[1] T. A. Cavalcanti, B. Shirinzadeh and S. Ikeda, "Nanorobot for Brain Aneurysm," Int. J. of Robot. Res., vol. 28, no. 4, pp. 558-570, 2009.

[2] B. J. Nelson, I. K. Kaliakatsos, and J. J. Abbott, "Microrobots for minimally invasive medicine," Annual Review of Bio.med. Eng., vol. 12 , no. 1 , pp. $55-85,2010$

[3] J. J. Abbott, K. E. Peyer, M. C. Lagomarsino, L. Zhang, L. X. Dong, I. K. Kaliakatsos, and B. J. Nelson, "How should microrobots swim?" Int. J. of Robot. Res., Jul. 2009.
[4] L. Zhang, K. E. Peyer, and B. J. Nelson, "Artificial bacterial flagella for micromanipulation," Lab on a Chip, vol. 10, no. 17, pp. 2203$2215,2010$.

[5] K. E. Peyer, L. Zhang, and B. J. Nelson, "Bio-inspired magnetic swimming microrobots for biomedical applications," Nanoscale, vol. 5, no. 4, pp. 1259-1272, 2013

[6] A. Evans and E. Lauga, "Propulsion by passive filaments and active flagella near boundaries," Phys. Rev. E, vol. 82, no. 4, p. 041915, Oct. 2010.

[7] L. Arcèse, M. Fruchard, and A. Ferreira, "Endovascular MagneticallyGuided Robots: Navigation Modeling and Optimization," IEEE Trans. Biomed. Eng., vol. 59, no. 4, pp. 977-987, 2012.

[8] S. Martel, J.-B. Mathieu, O. Felfoul, A. Chanu, E. Aboussouan, S. Tamaz, P. Pouponneau, L. Yahia, G. Beaudoin, G. Soulez, and M. Mankiewicz, "Automatic navigation of an untethered device in the artery of a living animal using a conventional clinical magnetic resonance imaging system," Applied Physics Letters, vol. 90, no. 11, p. $114105,2007$.

[9] Y. Sun, S. N. Fry, D. Potasek, D. J. Bell, and B. J. Nelson, "Characterizing fruit fly flight behavior using a microforce sensor with a new comb-drive configuration," J. of Microelectromechanical Systems, vol. 14, no. 1, pp. 4-11, 2005.

[10] W. A. Ducker, T. J. Senden, and R. M. Pashley, "Measurement of forces in liquids using a force microscope," Langmuir, vol. 8, no. 7, pp. 1831-1836, 1992.

[11] M. E. Fauver, D. L. Dunaway, D. H. Lilienfeld, H. G. Craighead, and G. H. Pollack, "Microfabricated cantilevers for measurement of subcellular and molecular forces," IEEE Trans. Biomed. Eng., vol. 45, no. 7, pp. 891-898, 1998.

[12] J. N. Fass and D. J. Odde, "Tensile force-dependent neurite elicitation via anti-1 integrin antibody-coated magnetic beads," Biophysical Journal, vol. 85, no. 1, pp. 623-636, 2003.

[13] X. Wang, G. Ananthasuresh, and J. P. Ostrowski, "Vision-based sensing of forces in elastic objects," Sensors and Actuators A: Physical, vol. 94, no. 3, pp. 142-156, 2001.

[14] M. A. Greminger and B. J. Nelson, "Vision-based force measurement," IEEE Trans. Pattern Anal. Machine Intell., vol. 26, no. 3, pp. 290-298, 2004

[15] O. Felfoul, J.-B. Mathieu, G. Beaudoin, and S. Martel, "In vivo mrtracking based on magnetic signature selective excitation," IEEE Trans. Med. Imag., vol. 27, no. 1, pp. 28-35, 2008.

[16] C. Bergeles, K. Shamaei, J. J. Abbott, and B. J. Nelson, "Singlecamera focus-based localization of intraocular devices," IEEE Trans. Biomed. Eng., vol. 57, no. 8, pp. 2064-2074, 2010.

[17] K. Belharet, D. Folio, and A. Ferreira, "Control of a magnetic microrobot navigating in microfluidic arterial bifurcations through pulsatile and viscous flow," in IEEE/RSJ Int. Conf. on Intel. Robots and Systems (IROS'2012), Vilamoura, Algarve, Portugal, Oct. 2012, pp. 2559-2564.

[18] G. Xu and Z. Zhang, Epipolar geometry in stereo, motion and object recognition: a unified approach. Springer, 1996, vol. 6.

[19] F. Chaumette and S. Hutchinson, "Visual servo control, part I: Basic approaches," IEEE Robot. Automat. Mag., vol. 13, no. 4, pp. 82-90, Dec. 2006.

[20] S. Hutchinson, G. Hager, and P. Corke, "A tutorial on visual servo control," IEEE Trans. Robot. Automat., vol. 12, no. 5, pp. 651-670, Oct. 1996.

[21] R. Tsai, "A versatile camera calibration technique for high-accuracy 3D machine vision metrology using off-the-shelf tv cameras and lenses," IEEE J. Robot. Automat., vol. 3, no. 4, pp. 323-344, Aug. 1987.

[22] J.-Y. Bouguet. (2004) Camera calibration toolbox for matlab. [Online]. Available: http://www.vision.caltech.edu/bouguetj/calib_doc/

[23] J. Oliensis, "A critique of structure-from-motion algorithms," Comp Vis. and Image Understanding, vol. 80, no. 2, pp. 172-214, 2000.

[24] L. Matthies, T. Kanade, and R. Szeliski, "Kalman filter-based algorithms for estimating depth in image sequences," Int. J. of Computer Vision, vol. 3, no. 3, pp. 209-238, 1989.

[25] S. Thrun, D. Fox, W. Burgard, and F. Dallaert, "Robust mote-carlo localization for mobile robots," Artifial Intelligence, vol. 128, no. 1-2, pp. 99-141, May 2001

[26] D. Folio and V. Cadenat, "Dealing with visual features loss during a vision-based task for a mobile robot," Int. J. of Optomechatronics, vol. 2, pp. 185-204, 2008 . 